\title{
Branes constrictions with White Dwarfs
}

\author{
Miguel A. García-Aspeitia ${ }^{1,2, a}$ \\ ${ }^{1}$ Consejo Nacional de Ciencia y Tecnología, Av, Insurgentes Sur 1582, Colonia Crédito Constructor, Del. Benito Juárez, \\ C.P. 03940 Mexico, D.F., Mexico \\ ${ }^{2}$ Unidad Académica de Física, Universidad Autónoma de Zacatecas, Calzada Solidaridad esquina con Paseo a la Bufa S/N, \\ C.P. 98060 Zacatecas, Mexico
}

Received: 7 September 2015 / Accepted: 21 October 2015 / Published online: 6 November 2015

(C) The Author(s) 2015. This article is published with open access at Springerlink.com

\begin{abstract}
We consider here a robust study of stellar dynamics for white dwarf stars with polytropic matter in the weakfield approximation using the Lane-Emden equation from the brane-world scenario. We also derive an analytical solution to the nonlocal energy density and show the behavior and sensitivity of these stars to the presence of extra dimensions. Similarly, we analyze stability and compactness, in order to show whether it is possible to agree with the conventional wisdom of white dwarfs dynamics. Our results predict an average value of the brane tension of $\langle\lambda\rangle \gtrsim 84.818 \mathrm{MeV}^{4}$, with a standard deviation $\sigma \simeq 82.021 \mathrm{MeV}^{4}$, which comes from a sample of dwarf stars, being weaker than other astrophysical observations but remaining higher than cosmological results provided by nucleosynthesis among others.
\end{abstract}

\section{Introduction}

Stellar astrophysics has been a cornerstone to demonstration of the predictive capabilities of the General Theory of Relativity (GR), describing high energy astrophysical phenomena such as white dwarfs and neutron stars, with unprecedented success [1-3]. One of the most important results in this vein is the Lane-Emden (LE) equation [4,5], which is a Newtonian approach to GR, under the assumption that the dwarf star is formed by polytropic matter; we remark that these types of stars are excellent high energy laboratories with which it is possible to test the phenomena described by GR and even to corroborate or refute our most plausible extensions [6-12].

Moreover, brane-world theory (for a good review see $[13,14])$ has been one of the most captivating extensions to GR, due to its theoretical predictions and its ability to solve fundamental phenomena such as the hierarchy problem, among others [15-17]. It is worth mentioning that the braneworld models have a very long tradition in the specialized

\footnotetext{
a e-mail: aspeitia@fisica.uaz.edu.mx
}

literature and their properties have been extensively studied under diverse circumstances, ranging from the cosmological scenarios [18-25] to the study of astrophysical models [6].

Following the conventional wisdom, it is possible to extend the classical astrophysics for polytropic stellar systems with the brane-worlds frame work. In this vein, many authors have been given the task of showing the different stellar behavior, studying stability, collapse [6-12] or the stellar dynamics in general [6-12].

With a view of this scenario, this paper is devoted to a study of the modifications of LE equation caused by the brane in the cases of a star with polytropic matter, it being our main goal to produce observational verifications in these systems. It is important to remark that here we have one of the most suitable signatures is the sensitivity of these kinds of stars to the corrections provided by brane theory, producing a new dynamics in energy density (or pressure) and in the effective mass; as well as the implementation of a new range of exclusion, where the star is dynamically unstable. From this new range, it is possible to propose a bound to the brane tension in order to avoid an unstable stellar configuration among other pathologies.

Before starting, we would like to mention here some experimental constraints on brane-world models, most of them concerning the so-called brane tension $\lambda$, which appears explicitly as a free parameter in the corrections of the gravitational equations mentioned above. As a first example we have the measurements on the deviations from Newton's law of the gravitational interaction at small distances. It is reported that no deviation is observed for distances $l \gtrsim 0.1 \mathrm{~mm}$, which then implies a lower limit on the brane tension in the model Randall-Sundrum II (RSII): $\lambda>1 \mathrm{TeV}^{4}$ [26,27]; it is important to mention that these limits do not apply to the two-branes case of the model Randall-Sundrum I (RSI) (see [14] for details). Astrophysical studies related with gravitational waves and stellar stability constrain the brane tension 
as $\lambda>5 \times 10^{8} \mathrm{MeV}^{4}[6-12,28,29]$, whereas the existence of black hole X-ray binaries suggests that $l \lesssim 10^{-2} \mathrm{~mm}$ $[14,30,31]$. Finally, from cosmological observations, the requirement of successful nucleosynthesis provides the lower limit $\lambda>1 \mathrm{MeV}^{4}$, which is a much weaker limit as compared to other experiments (other cosmological tests can be found in $[18-25,32,33])$.

We divide this paper in the following sections: Sect. 2 is dedicated to showing the equations of motion for a stellar structure, showing the modified Tolman-OppenheimerVolkoff (TOV) equation and the respective conservation equations; considering always the regularity of the functions and maintaining a Schwarzschild stellar exterior [6-12]. In Sect. 3 we derive the LE and mass equations, based on a set of minimal assumptions which are in concordance with the current studies of stellar dynamics. Also, an analytical form of the nonlocal energy density is derived which essentially is a function of the polytropic constant and the interior central energy density of the star. In Sect. 4 the initial conditions are imposed and we generate numerical solutions to the LE and mass equations for the case with a polytropic index of $n=3$, related with white dwarf stars. Finally in Sect. 5 we give some conclusions and make important remarks.

Henceforth we will use units in which $\hbar=c=1$, unless explicitly stated otherwise.

\section{Equations of motion}

Let us start by writing the equations of motion for stellar stability in a brane embedded in a five-dimensional bulk according to the RSII model [16]. Following an appropriate computation (for details see [14,34]), it is possible to demonstrate that the modified four-dimensional Einstein equations can be written as

$G_{\mu \nu}+\xi_{\mu \nu}+\Lambda_{(4)} g_{\mu \nu}=\kappa_{(4)}^{2} T_{\mu \nu}+\kappa_{(5)}^{4} \Pi_{\mu \nu}+\kappa_{(5)}^{2} F_{\mu \nu}$,

where $\kappa_{(4)}$ and $\kappa_{(5)}$ are, respectively, the four- and fivedimensional coupling constants, which are related in the form $\kappa_{(4)}^{2}=8 \pi G_{N}=\kappa_{(5)}^{4} \lambda / 6$, where $\lambda$ is defined as the brane tension, and $G_{N}$ is the Newton constant. For purposes of simplicity, we will not consider bulk matter, which translates into $F_{\mu \nu}=0$, and discard the presence of the fourdimensional cosmological constant, $\Lambda_{(4)}=0$, as we do not expect it to have an important effect at astrophysical scales (for a recent discussion as regards the cosmological constant see [35]). Additionally, we will neglect any nonlocal energy flux, which is allowed by the static spherically symmetric solutions we will study below [6-12].

The energy-momentum tensor, the quadratic energymomentum tensor, and the Weyl (traceless) contribution have the explicit forms

$$
\begin{aligned}
T_{\mu \nu} & =\rho u_{\mu} u_{\nu}+p h_{\mu \nu}, \\
\Pi_{\mu \nu} & =\frac{1}{12} \rho\left[\rho u_{\mu} u_{\nu}+(\rho+2 p) h_{\mu \nu}\right], \\
\xi_{\mu \nu} & =-\frac{\kappa_{(5)}^{4}}{\kappa_{(4)}^{4}}\left[\mathcal{U} u_{\mu} u_{\nu}+\mathcal{P} r_{\mu} r_{\nu}+\frac{h_{\mu \nu}}{3}(\mathcal{U}-\mathcal{P})\right] .
\end{aligned}
$$

Here, $p$ and $\rho$ are, respectively, the pressure and energy density of the stellar matter of interest, $\mathcal{U}$ is the nonlocal energy density, and $\mathcal{P}$ is the nonlocal anisotropic stress. Also, $u_{\alpha}$ is the four-velocity (which also satisfies the condition $\left.g_{\mu \nu} u^{\mu} u^{\nu}=-1\right), r_{\mu}$ is an unit radial vector, and $h_{\mu \nu}=g_{\mu \nu}+u_{\mu} u_{\nu}$ is the projection operator orthogonal to $u_{\mu}$.

Spherical symmetry indicates that the metric can be written

$$
\mathrm{d} s^{2}=-B(r) \mathrm{d} t^{2}+A(r) \mathrm{d} r^{2}+r^{2}\left(\mathrm{~d} \theta^{2}+\sin ^{2} \theta \mathrm{d} \varphi^{2}\right) .
$$

If we define the reduced Weyl functions $\mathcal{V}=6 \mathcal{U} / \kappa_{(4)}^{4}$, and $\mathcal{N}=4 \mathcal{P} / \kappa_{(4)}^{4}$, then the equations of motion for a relativistic star in the brane are

$\mathcal{M}^{\prime}=4 \pi r^{2} \rho_{\text {eff }}$,

$p^{\prime}=-\frac{G_{N}}{r^{2}} \frac{4 \pi p_{\text {eff }} r^{3}+\mathcal{M}}{1-2 G_{N} \mathcal{M} / r}(p+\rho)$,

$\mathcal{V}^{\prime}+3 \mathcal{N}^{\prime}=-\frac{2 G_{N}}{r^{2}} \frac{4 \pi p_{\text {eff }} r^{3}+\mathcal{M}}{1-2 G_{N} \mathcal{M} / r}(2 \mathcal{V}+3 \mathcal{N})$

$$
-\frac{9}{r} \mathcal{N}-3(\rho+p) \rho^{\prime}
$$

where a prime indicates a derivative with respect to $r, A(r)=$ $\left[1-2 G_{N} \mathcal{M}(r) / r\right]^{-1}$, and the effective energy density and pressure, respectively, are given by

$\rho_{\text {eff }}=\rho\left(1+\frac{\rho}{2 \lambda}\right)+\frac{\mathcal{V}}{\lambda}$,

$p_{\text {eff }}=p\left(1+\frac{\rho}{\lambda}\right)+\frac{\rho^{2}}{2 \lambda}+\frac{\mathcal{V}}{3 \lambda}+\frac{\mathcal{N}}{\lambda}$.

Even though we will not consider exterior solutions, we must anyway take into account the information provided by the Israel-Darmois (ID) matching condition, which for the case of our study can be written as [6-12]

$$
(3 / 2) \rho^{2}(R)+\mathcal{V}^{-}(R)+3 \mathcal{N}^{-}(R)=\mathcal{V}^{+}(R)+3 \mathcal{N}^{+}(R),
$$

where the superscript $-(+)$ denotes the interior (exterior) values of the different quantities at the surface of the star, and we also assumed that $\rho(r>R)=0$.

A desirable property we want in our solutions is a Schwarzschild exterior, which can easily be accomplished under the boundary conditions $\mathcal{V}^{+}(R)=0=\mathcal{N}^{+}(R)$, as for them the simplest solution that arises from Eq. (4c) is the 
trivial one: $\mathcal{V}(r \geq R)=0=\mathcal{N}(r \geq R)$. Thus, for the purposes of this paper, we will refer hereafter to the restricted ID matching condition given by

$(3 / 2) \rho^{2}(R)+\mathcal{V}^{-}(R)+3 \mathcal{N}^{-}(R)=0$.

Just for completeness, we note that the exterior solutions of the metric functions are given by the well-known expressions $B(r)=A^{-1}(r)=1-2 G_{N} M / r$.

Finally, an important feature is that the only interior solution of the nonlocal anisotropic stress under the conditions of a Schwarzschild exterior, and for a non-constant density with $\rho(R)=0$, which are the conditions we expect to have in realistic stars, is the trivial one: $\mathcal{N}(r) \equiv 0$ (see [6-12] for details). This implies that Eq. (7) can be written

$-(3 / 2) \rho^{2}(R)=\mathcal{V}^{-}(R)$,

with the aim of maintaining a Schwarzschild exterior.

\section{The modified Lane-Emden equation}

In principle, we should just numerically evolve Eq. (4), but as we have to deal with weak gravity we find it more appropriate to evolve the weak-field limit of such system of equations, which by the way provides important technical simplifications that let us to have more physical insight. In order to get a star as real as possible and find the LE equation in the case of brane stars, we start imposing the following minimal conditions:

(a) The radius $R$ is fixed, with $\rho(r)=0$ for $r>R$ [6-12].

(b) The pressure vanishes at the surface and in the exterior of the star, and the $p(r)=0$ for $r \geq R$ [6-12].

(c) The star is described by the polytropic equation $p=$ $K \rho^{(1+n) / n}$, where $n$ is the polytropic index with $n \geq 0$.

(d) The pressure is negligible compared with the energy density $p \ll \rho$.

(e) We assume the relation $4 \pi r^{3} p_{\text {eff }} \ll \mathcal{M}$ between the effective variables.

(f) The gravitational potential in terms of the effective mass is negligible, $2 G_{N} \mathcal{M} / r \ll 1$.

Conditions (a) and (b) are conventional wisdom, being physically reasonable assumptions for stellar configurations; both conditions are not imposed in the dynamical equations, however, we expect that they are satisfied in order to obtain a real star. For instance, in the case of condition (c) we propose a polytropic equation, which is the most similar component to a real star, condition (d) is necessary for the Newtonian approach, conditions (e) and (f) are similarly necessary for the Newtonian approach but also important to make the comparison between the terms which generate the effective pressure and effective mass.

To begin with, we observe that under conditions (c)-(f) and from Eq. (4b) we have

$r^{2} p^{\prime}=-G_{N} \mathcal{M} \rho$

differentiating we find

$\frac{d}{\mathrm{~d} r}\left(\frac{r^{2}}{\rho} \frac{\mathrm{d} p}{\mathrm{~d} r}\right)=-4 \pi G_{N} \rho_{\mathrm{eff}}$.

Considering the following change of variables $[4,5]$ :

$r=\left(\frac{K(n+1)}{4 \pi G_{N}}\right)^{1 / 2} \rho(0)^{(1-n) / 2 n} \zeta$,

$\rho=\rho(0) \theta^{n}, \quad p=K \rho(0)^{(n+1) / n} \theta^{n+1}$,

and substituting in Eq. (10), it is possible to write the LE equation modified by the presence of branes,

$\frac{1}{\zeta^{2}} \frac{d}{\mathrm{~d} \zeta} \zeta^{2} \frac{\mathrm{d} \theta}{\mathrm{d} \zeta}+\theta^{n}+\bar{\rho}\left(\theta^{2 n}+\overline{\mathcal{V}}(\theta)_{n}\right)=0$,

where $\overline{\mathcal{V}}(\theta)_{n} \equiv 2 \mathcal{V}(\theta)_{n} / \rho(0)^{2}, \bar{\rho} \equiv \rho(0) / 2 \lambda$. In addition, from Eq. (4a) and the renaming of variables (11), we obtain the dimensionless mass equation:

$\frac{\mathrm{d} \overline{\mathcal{M}}}{\mathrm{d} \zeta}-\theta^{n}-\bar{\rho}\left(\theta^{2 n}+\overline{\mathcal{V}}(\theta)_{n}\right)=0$,

or in quadrature

$\overline{\mathcal{M}}=\int_{0}^{\xi_{R}} \theta^{n} \mathrm{~d} \zeta+\bar{\rho} \int_{0}^{\xi_{R}}\left(\theta^{2 n}+\overline{\mathcal{V}}(\theta)_{n}\right) \mathrm{d} \zeta$

where $\overline{\mathcal{M}} \equiv G_{N}^{3 / 2} \rho(0)^{-(3-n) / 2 n} \mathcal{M} /\left((4 \pi)^{1 / 3} K(n+1)\right)^{3 / 2}$. It is straightforward to see that the non-brane limit is recovered when $\bar{\rho} \rightarrow 0$, in Eqs. (12)-(14). The opposite case is the brane domination terms limit, when $\bar{\rho} \gg 1$, leading to the following equations of motion:

$\frac{1}{\zeta^{2}} \frac{d}{\mathrm{~d} \zeta} \zeta^{2} \frac{\mathrm{d} \theta}{\mathrm{d} \zeta}+\bar{\rho}\left[\theta^{2 n}+\overline{\mathcal{V}}(\theta)_{n}\right]=0$,

$\frac{\mathrm{d} \overline{\mathcal{M}}}{\mathrm{d} \zeta}-\bar{\rho}\left[\theta^{2 n}+\overline{\mathcal{V}}(\theta)_{n}\right]=0$.

Now, it is necessary to find the explicit functional form of the nonlocal energy density from the conservation equation (4c), and with the help of conditions (d)-(f) we have

$r^{2} \mathcal{V}^{\prime}+4 G_{N} \mathcal{M V}=-3(\rho+p) r^{2} \rho^{\prime}$ 
differentiating and rearranging with the use of Eqs. (11) we obtain in general the following first order differential equation:

$\frac{\mathrm{d} \overline{\mathcal{V}}_{n}}{\mathrm{~d} \theta}-\chi_{n} \overline{\mathcal{V}}_{n}=-6 n\left[\theta^{2 n-1}+\frac{\chi_{n}}{4(n+1)} \theta^{2 n}\right]$,

whose solution can be computed through the following integral:

$$
\begin{aligned}
\overline{\mathcal{V}}_{n} & =-6 n \exp \left(\chi_{n} \theta\right) \int\left[\theta^{2 n-1}+\frac{\chi_{n}}{4(n+1)} \theta^{2 n}\right] \\
& \times \exp \left(-\chi_{n} \theta\right) \mathrm{d} \theta+\mathcal{C} \exp \left(\chi_{n} \theta\right),
\end{aligned}
$$

where we have also introduced the dimensionless quantities, $\chi_{n} \equiv 4(n+1) K \rho(0)^{1 / n}$ for $n \neq 0, \mathcal{C}$ is an integration constant and $\overline{\mathcal{V}}_{n}$ is a function of $\theta$, which in turn is a function of $r$. The solution of the previous differential equation, without loss of generality, can be written as

$$
\begin{aligned}
\overline{\mathcal{V}}(\theta)_{n}= & \frac{6 n}{\chi_{n}^{2 n}} \exp \left(\chi_{n} \theta\right)\left\{\Gamma\left(2 n, \chi_{n} \theta\right)+\frac{\Gamma\left(2 n+1, \chi_{n} \theta\right)}{4(n+1)}\right\} \\
& +\mathcal{C}_{1} \exp \left(\chi_{n} \theta\right)
\end{aligned}
$$

for $n \geq 1 / 2, \Gamma(x, y)$ being the incomplete gamma function. Also we have

$$
\begin{aligned}
\overline{\mathcal{V}}(\theta)_{n}= & 6 n \exp \left(\chi_{n} \theta\right)\left\{\frac{\Gamma\left(2(1-n), \chi_{n} \theta\right)}{\chi_{n}^{2(1-n)}}+\frac{\Gamma\left(2 n+1, \chi_{n} \theta\right)}{4(n+1) \chi_{n}^{2 n}}\right\} \\
& +\mathcal{C}_{2} \exp \left(\chi_{n} \theta\right),
\end{aligned}
$$

for $0<n<1 / 2$. In both cases, $\mathcal{C}_{1}$ and $\mathcal{C}_{2}$ are integration constants associated with the initial condition. Notice that, in principle, the modified LE equation does not accept solutions for $n=0$, due to the divergence of the nonlocal energy density $\overline{\mathcal{V}}_{n}$; this would imply an unstable and non-compact stellar configuration to be a prediction of this model.

Particularly, low energy stars like dwarf stars can be modeled in this context and now we are in a position to determine how the brane effects provide the interior of a star with extra dynamics. It is important to mention that white dwarfs can be modeled by the polytropic index $n=3$, and neutron stars by polytropes with an index in the range $n=0.5-1$. However, in the case of neutron stars the weak-field approximation is not sufficient to make a general description of these stars; it is necessary to add the corrections provided by GR with the full modified TOV equation.

\section{Numerical solutions for dwarf stars}

Let us start studying a dwarf star using the modified LE equation; we observe from Eq. (12) that the free parameters are $\bar{\rho}$ and $\chi_{n}$, related with the central energy density of the star, the brane tension, and the polytropic constant.

Our analysis shows that the central energy density and the polytropic constant are redundant, because in particular they depend on the characteristics of each star; then we fix by hand the values of $\chi_{n}$, where are encoded by both parameters. In this case, we board the region $\chi_{3}=10$, due to the orders of magnitude being greater, causing divergences which imply non-compact configurations. This results in the dependence $K=5 \rho(0)^{-1 / 3} / 8$. Therefore, we only explore the limit case, when the minimal requirements (a)-(f) are fulfilled.

\subsection{Physical initial conditions}

Some physical initial conditions for the star are important; for this reason we start showing the equations of kinetic energy density and pressure of electrons of the dwarf star $[4,5]$ :

$$
\begin{aligned}
& e=\frac{8 \pi}{(2 \pi)^{3}} \int_{0}^{k_{F}}\left[\left(k^{2}+m_{e}^{2}\right)^{1 / 2}-m_{e}\right] k^{2} \mathrm{~d} k, \\
& p=\frac{8 \pi}{3(2 \pi)^{3}} \int_{0}^{k_{F}} \frac{k^{2}}{\left(k^{2}+m_{e}^{2}\right)^{1 / 2}} k^{2} \mathrm{~d} k,
\end{aligned}
$$

where the momenta are between $k$ and $k+\mathrm{d} k, k_{F}$ being the maximum momentum and $m_{e}$ being related with the electron mass. From Eqs. (21) and (22), we obtain for the dwarf stars with index $n=3$ the following conditions:

$$
\begin{aligned}
e & =3 p, \quad p=\frac{1}{12 \pi^{2}}\left(\frac{3 \pi^{2} \rho}{m_{N} \mu}\right)^{4 / 3}, \\
K & =\frac{1}{12 \pi^{2}}\left(\frac{3 \pi^{2}}{m_{N} \mu}\right)^{4 / 3},
\end{aligned}
$$

where $\mu$ is the number of nucleons per electron and $m_{N}$ is the nucleon mass $[4,5]$.

\subsection{Results of the numerical solutions}

To begin with, we show the numerical solutions implemented for dwarf stars showing the behavior of energy density and mass profiles in Fig. 1, top and bottom. We implement the usual initial conditions: $\theta(0)=1, \mathrm{~d} \theta(0) / \mathrm{d} \zeta=0$ and $\overline{\mathcal{M}}(0)=0$, for $n=3$, as in the textbook case [4,5] and $\overline{\mathcal{V}}(0)=0$ considering an inward integration.

We start showing the non-brane case as a benchmark, adding first only the quadratic part of the energy-momentum tensor. Under this assumption, we predict a lower energy density compared with the non-brane case (see Fig. 1, top). Clearly, the stellar configuration is more massive for a similar radius to the previous case (see Fig. 1, bottom). Also, we present the compactness plot (see Fig. 2), which shows the different behaviors with different values of the brane terms. It is obvious how we have a most compact configuration when the presence of the quadratic terms predicted by branes plays 
an important role. Clearly, this is an incomplete analysis due to lack of Weyl terms, however, in the following, we took on the task of presenting the nonlocal terms.

When we turn on the Weyl terms, these cause higher energy densities and smaller masses in comparison with the case of non-branes, while we increase the presence of extra terms; the effects are accentuated, causing a non-compact configuration, i.e., conditions (a) and (b) are not fulfilled. In this sense, one may note that $\bar{\rho}=0.016$ is the higher bound to a stable stellar configuration; we notice that when we exceed this bound we have an unstable star, implying a non-real stellar structure (see Fig. 1, top and bottom, and Fig. 2).

Considering a stable star configuration which meets the minimal condition (a) $-(\mathrm{g})$, it is possible to find the brane tension bound as $\lambda \gtrsim 29.585 \rho(0)$, where $\rho(0)$ corresponds to the central energy density of the dwarf star. With the aim of comparing with astrophysical data, in Table 1 we show ten dwarf stars collected by the catalogs reported in Refs. [36-38], mainly emphasizing the values of mass, radius, and central density; then for the samples of Table 1 and under the assumption that the observed white dwarfs must belong to a family of equilibrium configuration without an anomalous behavior, the average value of the brane tension must be $\langle\lambda\rangle \gtrsim 84.818 \mathrm{MeV}^{4}$, with a standard deviation $\sigma \simeq 82.021 \mathrm{MeV}^{4}$, showing too much dispersion in the set of the sample. This is attributed to the marked differences between the dwarf stars. In addition, notice how our results are weaker than other astrophysical data [6-12]; however, it remains above the levels provided by cosmological bounds like nucleosynthesis [18-25,32,33].

\section{Conclusions and remarks}

The presented analysis of a weak field maintaining the brane terms, conducted by using the LE equation, shows the new behavior of the density, the mass, and the compactness of stars with polytropic matter. The research developed in this paper shows how dwarf stars are sensitive to the Weyl terms, causing a non-compact configuration under particular conditions, implying a non-real star. It should be mentioned that only the existence of quadratic terms in the energy momentum tensor shows a less dense and more massive star compared to the non-brane case. In general, when we turn on also the Weyl contributions, the star's model rather suggests a behavior of higher energy density and lower mass, beyond standard GR, which is discussed in 4.2. Significantly, there is a physical limit to the parameters $\bar{\rho}$ and $\chi_{n}$ (see Figs. $1,2)$ such that one meets the minimum requirements for a stable star, which in this case must be $\lambda \gtrsim 29.585 \rho(0)$
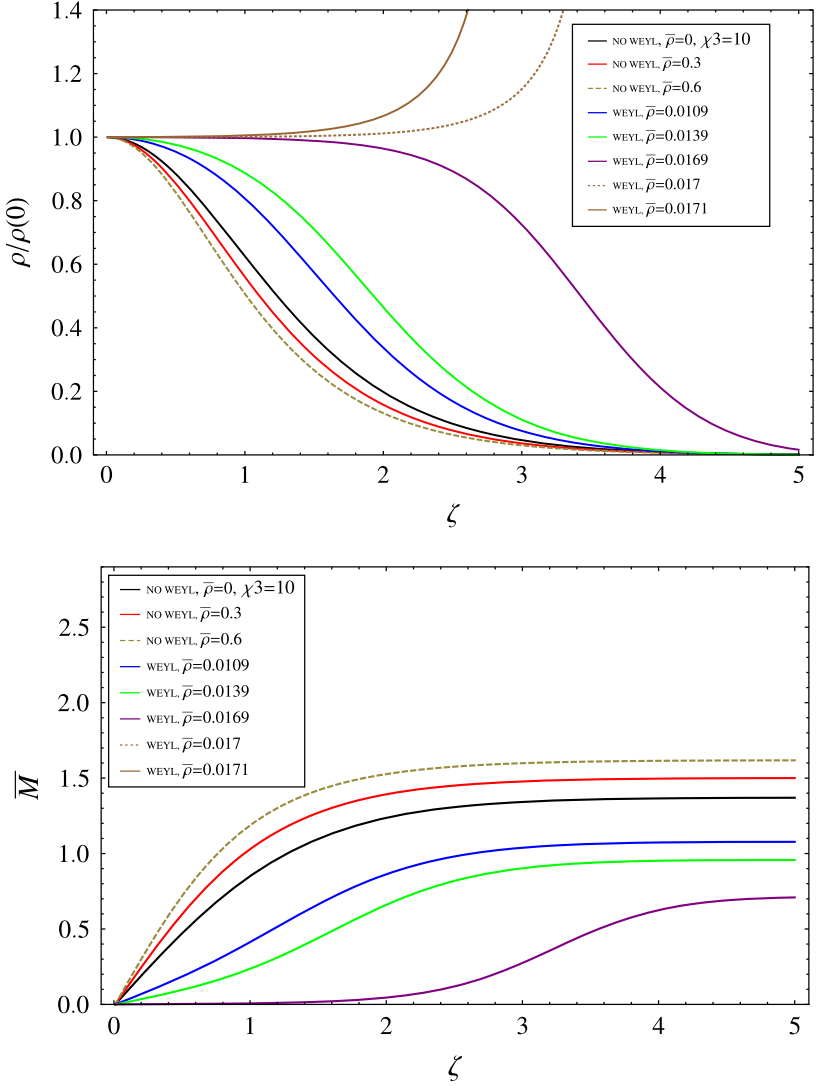

Fig. 1 Numerical solution of Eqs. (12)-(14) for white dwarfs with polytropic index $n=3$. Here we show the energy density (top) and effective mass (bottom) of the white dwarf stars. Notice the sensitivity to the term $\bar{\rho}$, when we turn on the Weyl terms. When Weyl terms are dominant, the stellar configuration is unstable, causing conditions (a) and (b) not to be fulfilled. See the text for more details

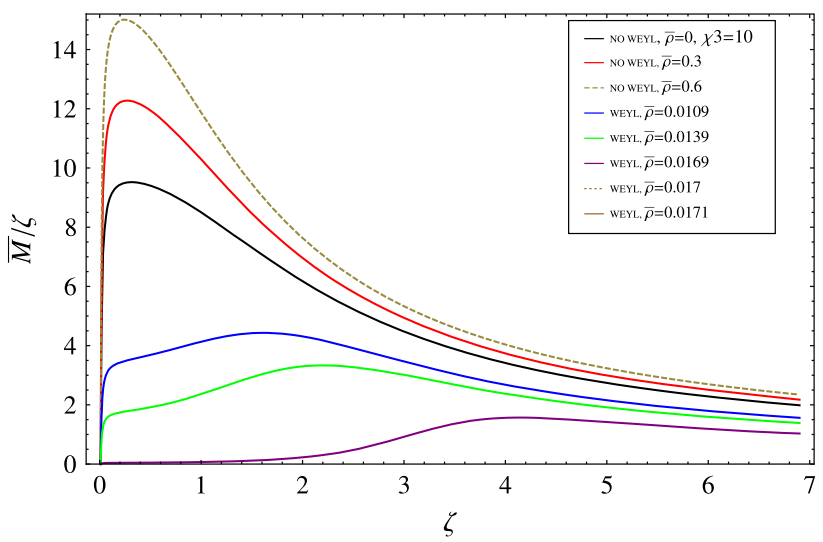

Fig. 2 Numerical solution of Eqs. (12)-(14) for white dwarfs with polytropic index $n=3$. Here we show the compactness $\overline{\mathcal{M}} / \zeta$ in the presence of Weyl terms and without. The presence of both terms generates a less compactness stellar configuration with a maximum displaced in comparison with the other cases. Notice how $\bar{\rho}=0.0169$ is the constriction for the plots. See the text for more details 
Table 1 From left to right the columns read: name of the star, mass in solar units $M_{\odot}$, radius in $R_{\odot}$, density as $\rho(0)=3 M / 4 \pi R^{3}$ in $\mathrm{MeV}^{4}$, and brane tension in $\mathrm{MeV}^{4}$ deduced from the constraint mentioned above; using a catalog of several white dwarfs reported in [36-38]

\begin{tabular}{|c|c|c|c|c|}
\hline White Dwarf & $\operatorname{Mass}\left(M_{\odot}\right)$ & Radius $\left(R_{\odot}\right)$ & $\rho(0)\left(\mathrm{MeV}^{4}\right)$ & $\lambda\left(\mathrm{MeV}^{4}\right)$ \\
\hline Sirius B & 1.034 & 0.0084 & 10.5993 & 313.588 \\
\hline Procyon B & 0.604 & 0.0096 & 4.1478 & 122.715 \\
\hline 40 Eri B & 0.501 & 0.0136 & 1.21009 & 35.801 \\
\hline EG 50 & 0.50 & 0.0104 & 2.70063 & 79.900 \\
\hline GD 140 & 0.79 & 0.0085 & 7.81565 & 231.232 \\
\hline CD-38 10980 & 0.74 & 0.01245 & 2.3298 & 68.928 \\
\hline W485A & 0.59 & 0.0150 & 1.06212 & 31.423 \\
\hline G154-B5B & 0.46 & 0.0129 & 1.3006 & 38.4793 \\
\hline LP $347-6$ & 0.56 & 0.0124 & 1.7827 & 52.7426 \\
\hline G181-B5B & 0.54 & 0.0125 & 1.6781 & 49.6479 \\
\hline WD1550+130 & 0.535 & 0.0211 & 0.3456 & 10.2266 \\
\hline Stein 2051B & 0.48 & 0.0111 & 2.13023 & 63.0229 \\
\hline G107-70AB & 0.65 & 0.0127 & 1.926 & 56.9807 \\
\hline L268-92 & 0.70 & 0.0149 & 1.28438 & 37.9984 \\
\hline G156-64 & 0.59 & 0.0110 & 2.69047 & 79.5976 \\
\hline
\end{tabular}

See the text for more details

and $K=5 /\left(8 \rho(0)^{1 / 3}\right)$. Taking astrophysical data of a sample of white dwarfs, it is possible to establish an average bound of the brane tension as shown in the previous section: $\langle\lambda\rangle \gtrsim 84.818 \mathrm{MeV}^{4}$, with a standard deviation $\sigma \simeq 82.021 \mathrm{MeV}^{4}$ and the average of the polytropic constant must be constrained as $\langle K\rangle \simeq 0.508 \mathrm{MeV}^{-4 / 3}$ with a standard deviation $\sigma \simeq 0.142 ; \mathrm{MeV}^{-4 / 3}$. It is important to remark how the previous values are necessary to fulfill the minimal requirements to obtain a stable star; i.e. a real stellar configuration.

It is important to clarify that nonlocal terms caused by Weyl terms are gravitons that escape to the fifth dimension, causing stars not to have a compact configuration as they begin to dominate. An excess of Weyl terms is the cause that it does not satisfy the conditions (a) and (b), as we show. In fact, the Weyl terms eventually generate a divergence for a given radius. However, we can use this disadvantage to quantify the minimum value required for the brane tension, which is shown in our conclusions.

In addition, it is worth mentioning that modifications to the LE equation prohibit the case $n=0$ (at least for the case where $\mathcal{C}_{1} \neq 0$ or $\mathcal{C}_{2} \neq 0$ ), for a stable stellar configuration unlike that predicted by the non-brane limit. This is due to the divergence in the $\chi_{n}$ term in the central energy density, causing conditions (a) and (b) not to be fulfilled.

Despite the fact that we are treating a weak gravitational limit and the brane effects are not accentuated strongly in the dynamics, it is possible to extract relevant information as regards the constraint of the brane tension, establishing an exclusion limit of the theory, taking as a premise the stability of the dwarf star. Strong evidence of branes can be found in the direct observation of the compactness of a dwarf star, when comparing the predictions of GR and branes, bearing in mind the technical challenges of this endeavor due to the subtle brane effects.

Finally, we suggest that studies of neutron stars can give us better constraints, and even evidence of the existence of extra dynamics which comes from brane theories. In the case of neutron stars, part of the machinery has been studied in Refs. [6-12], still as the most general way to treat this type of stars in a strong gravitational field. However, this is work that will be presented elsewhere.

Acknowledgments The author acknowledge the suggestions of the anonymous referee and the enlightening conversation with F. Linares. Also the author is grateful for the support provided by SNI-México, CONACyT research fellowship and Instituto Avanzado de Cosmología (IAC) collaborations.

Open Access This article is distributed under the terms of the Creative Commons Attribution 4.0 International License (http://creativecomm ons.org/licenses/by/4.0/), which permits unrestricted use, distribution, and reproduction in any medium, provided you give appropriate credit to the original author(s) and the source, provide a link to the Creative Commons license, and indicate if changes were made.

Funded by SCOAP 3 .

\section{References}

1. S. Chandrasekhar, The Mathematical Theory of Black Holes (Oxford University Press, Oxford, 1985)

2. R.C. Tolman, Relativity, Thermodynamics, and Cosmology (Dover Publications. com, Mineola, 1987)

3. J.R. Oppenheimer, G.M. Volkoff, Phys. Rev. 55, 374 (1939) 
4. S. Chandrasekhar, An Introduction to the Study of Stellar Structure, vol. 2 (Dover Publications. com, Mineola, 1958)

5. S. Weinberg, Gravitation and Cosmology: Principles and Applications of the General Theory of Relativity, vol. 1 (Wiley, New York, 1972)

6. C. Germani, R. Maartens, Phys. Rev. D 64, 124010 (2001)

7. J. Ovalle, F. Linares, A. Pasqua, A. Sotomayor, Class. Quantum Gravity 30, 175019 (2013). arXiv:1304.5995 [gr-qc]

8. J. Ovalle, F. Linares, Phys. Rev. D 88, 104026 (2013). arXiv:1311.1844 [gr-qc]

9. J. Ovalle, L. Gergely, R. Casadio (2014). arXiv:1405.0252 [gr-qc]

10. M.A. Garcia-Aspeitia, M.J. Reyes-Ibarra, C. Ortiz, J. LopezDominguez, S. Hinojosa-Ruiz (2014). arXiv:1412.3496 [gr-qc]

11. M.A. García-Aspeitia, L.A. Ureña Lopez, Class. Quantum Gravity 32, 025014 (2015). arXiv:1405.3932 [gr-qc]

12. F.X. Linares, M.A. García-Aspeitia, L.A. Ureña, López, Phys. Rev. D 92, 024037 (2015)

13. A. Perez-Lorenzana, J. Phys. Conf. Ser. 18, 224 (2005). arXiv:hep-ph/0503177

14. R. Maartens, K. Koyama, Living Rev. Rel. 13, 5 (2010). arXiv:1004.3962 [hep-th]

15. L. Randall, R. Sundrum, Phys. Rev. Lett. 83, 3370 (1999). arXiv:hep-ph/9905221

16. L. Randall, R. Sundrum, Phys. Rev. Lett. 83, 4690 (1999). arXiv:hep-th/9906064

17. S.T. McWilliams, Phys. Rev. Lett. 104, 141601 (2010)

18. R. Maartens, Phys. Rev. D 62, 084023 (2000). arXiv:hep-th/0004166

19. P. Brax, C. van de Bruck, A.-C. Davis, Rep. Prog. Phys. 67, 2183 (2004). arXiv:hep-th/0404011

20. P. Brax, C. van de Bruck, Class. Quantum Gravity 20, R201 (2003). arXiv:hep-th/0303095

21. M.A.G. Aspeitia, T. Matos, Gen. Relativ. Gravit. 43, 315 (2011). arXiv:0906.3278 [gr-qc]
22. M.A. Garcia-Aspeitia, J. Magana, T. Matos, Gen. Relativ. Gravit. 44, 581 (2012). arXiv:1102.0825 [gr-qc]

23. L.B. Castro, M.D. Alloy, D.P. Menezes, JCAP 1408, 047 (2014). arXiv:1403.1099 [nucl-th]

24. R. Casadio, J. Ovalle, Phys. Lett. B 715, 251 (2012). arXiv:1201.6145 [gr-qc]

25. R. Casadio, J. Ovalle, Gen. Relativ. Gravit. 46, 1669 (2014). arXiv: 1212.0409 [gr-qc]

26. D. Kapner, T. Cook, E. Adelberger, J. Gundlach, B.R. Heckel et al., Phys. Rev. Lett. 98, 021101 (2007). arXiv:hep-ph/0611184

27. S. Alexeyev, K. Rannu, P. Dyadina, B. Latosh, S. Turyshev (2015). doi:10.7868/S0044451015060051. arXiv:1501.04217v1

28. J. Sakstein, B. Jain, V. Vikram, Int. J. Mod. Phys. D 23, 1442002 (2014). arXiv:1409.3708 [astro-ph.CO]

29. M.A. Garcfa-Aspeitia, Rev. Mex. Fis. 60, 205 (2014). arXiv:1306.1283 [gr-qc]

30. H. Kudoh, T. Tanaka, T. Nakamura, Phys. Rev. D 68, 024035 (2003). arXiv:gr-qc/0301089

31. M. Cavaglia, Int. J. Mod. Phys. A 18, 1843 (2003). arXiv:hep-ph/0210296

32. R. Holanda, J. Silva, F. Dahia, Class. Quantum Gravity 30, 205003 (2013). arXiv:1304.4746 [astro-ph.CO]

33. J.D. Barrow, R. Maartens, Phys. Lett. B 532, 153 (2002). arXiv:gr-qc/0108073

34. T. Shiromizu, K. Maeda, M. Sasaki, Phys. Rev. D 62, 024012 (2000)

35. V. Pavlidou, T.N. Tomaras (2013). arXiv:1310.1920 [astro-ph.CO]

36. S. Balberg, S.L. Shapiro, arXiv preprint (2000). arXiv:astro-ph/0004317

37. H.L. Shipman, J. Provencal, E. Høg, P. Thejll, Astrophys. J. Lett. 488, L43 (1997)

38. J.B. Holberg, M. Barstow, F. Bruhweiler, A. Cruise, A. Penny, Astrophys. J. 497, 935 (1998) 